\title{
Preoperative plasma growth-differentiation factor-15 for prediction of acute kidney injury in patients undergoing cardiac surgery
}

Matthias Heringlake ${ }^{1 * \dagger} \mathbb{D}$, Efstratios I. Charitos ${ }^{2 \dagger}$, Kira Erber ${ }^{1}$, Astrid Ellen Berggreen ${ }^{1}$, Hermann Heinze and Hauke Paarmann ${ }^{3}$

\begin{abstract}
Background: Growth-differentiation factor-15 (GDF-15) is an emerging humoral marker for risk stratification in cardiovascular disease. Cardiac-surgery-associated acute kidney injury (CSA-AKI), an important complication in patients undergoing cardiac surgery, is associated with poor prognosis. The present secondary analysis of an observational cohort study aimed to determine the role of GDF-15 in predicting CSA-AKI compared with the Cleveland-Clinic Acute Renal Failure (CC-ARF) score and a logistic regression model including variables associated with renal dysfunction.

Methods: Preoperative plasma GDF-15 was determined in 1176 consecutive patients undergoing elective cardiac surgery. Patients with chronic kidney disease stage 5 were excluded. AKI was defined according to KidneyDisease-Improving-Global-Outcomes (KDIGO) - creatinine criteria. The following variables were screened for association with development of postoperative AKl: age, gender, additive Euroscore, serum creatinine, duration of cardiopulmonary bypass, duration of surgery, type of surgery, total circulatory arrest, preoperative hemoglobin, preoperative oxygen-supplemented cerebral oxygen saturation, diabetes mellitus, hemofiltration during ECC, plasma GDF-15, high sensitivity troponin T (hsTNT), and N-terminal prohormone of B-type natriuretic peptide (NTproBNP).
\end{abstract}

Results: There were 258 patients (21.9\%) with AKI (AKI stage 1 (AKI-1), $n=175$ (14.9\%); AKI-2, $n=6$ (0.5 \%); AKI-3, $n=77(6.5 \%)$ ). The incidence of AKI-1 and AKI-3 increased significantly from the lowest to the highest tertiles of GDF-15. In logistic regression, preoperative GDF-15, additive Euroscore, age, plasma creatinine, diabetes mellitus, and duration of cardiopulmonary bypass were independently associated with AKI. Inclusion of GDF-15 in a logistic regression model comprising these variables significantly increased the area under the curve (AUC 0.738 without and 0.750 with GDF-15 included) and the net reclassification ability to predict AKI. Comparably, in receiver operating characteristic analysis the predictive capacity of the CC-ARF score (AUC 0.628) was improved by adding GDF-15 (AUC 0.684) but this score also had lower predictability than the logistic regression model. In random forest analyses the predictive capacity of GDF-15 was especially pronounced in patients with normal plasma creatinine.

Conclusion: This suggests that preoperative plasma GDF-15 independently predicts postoperative AKI in patients undergoing elective cardiac surgery and is particularly helpful for risk stratification in patients with normal creatinine.

Trial registration: NCT01166360 on July 20, 2010.

Keywords: Cardiac surgery, Acute kidney injury, Biomarkers, Growth-differentiation factor-15, Cleveland Clinic Acute Renal Failure score, Euroscore

\footnotetext{
* Correspondence: Heringlake@t-online.de

${ }^{\dagger}$ Equal contributors

'Department of Anesthesiology and Intensive Care Medicine, University of

Lübeck, Ratzeburger Allee 160, D - 23538 Luebeck, Germany

Full list of author information is available at the end of the article
} 


\section{Background}

Cardiac-surgery-associated acute kidney injury (CSAAKI) is an important and frequent complication in patients undergoing cardiac surgery and associated with increased morbidity and short-term and long-term mortality [1]. The incidence of CSA-AKI is variable and depends on the definition used, but has been reported to be as high as $40 \%$ according to the Acute Kidney Injury Network (AKIN) criteria [2]. CSA-AKI requiring temporary renal replacement therapy occurs in up to $30 \%$ of patients and has been associated with a mortality rate up to $60 \%[1,3]$.

No specific treatment for the prevention of CSA-AKI is available [4]. This may be related to the multifactorial pathophysiology of this complication [5], including postoperative factors that are difficult to predict preoperatively [6], but also to the fact that sparse modalities for preoperative risk stratification are available and that commonly used risk scores have variable prognostic utility in this regard [7]. However, preoperative identification of patients with a high risk of developing CSA-AKI is a prerequisite for developing strategies to ameliorate or prevent perioperative renal injury.

Very recently, two studies in 32 and 134 patients, respectively, provided evidence that the preoperative plasma level of the hormone growth-differentiation factor-15 (GDF-15) predicts postoperative renal injury $[8,9]$ in patients undergoing coronary artery bypass graft (CABG). We have previously shown that the preoperative plasma concentration of GDF-15 is an independent predictor of morbidity and short-term and long-term mortality in patients undergoing cardiac surgery [10]. The present study aims to confirm the findings of the pilot studies $[8,9]$ in a larger and heterogenous patient cohort to determine if this hormone may also be used for assessing the risk of developing AKI in this population.

\section{Methods}

The present study is a secondary analysis of a large prospective observational cohort study analyzing the prognostic relevance of preoperative cerebral oxygen saturation and markers of cardiopulmonary dysfunction with respect to clinical outcomes in patients undergoing cardiac surgery [10, 11]. In total 2009 patients were screened during the study period between January and December 2008 and April to December 2009. There were 5 patients who refused to participate in the study, and 76 patients had their surgery cancelled. Complete datasets including GDF-15 measurements were available from 1458 patients and used for the previously published analyses [10]. Excluding emergency patients, off-pump revascularization, interventional procedures, and patients with chronic kidney disease stage 5, plasma samples for determination of plasma
GDF-15 were available from 1176 consecutive patients undergoing elective cardiac surgery, and these were used for the present analysis.

The primary objective was to determine the relationship between preoperative plasma GDF-15 and AKI [12] in comparison with the Cleveland Clinic acute renal failure (CC-ARF) score [13] and a comprehensive logistic regression model based on variables typically associated with AKI in patients undergoing cardiac surgery, to investigate whether GDF levels can further improve risk stratification for AKI.

Plasma samples for determination of GDF-15 were taken immediately preoperatively (before induction of anesthesia) and determined as described recently [10]. Plasma was separated and stored at $-80{ }^{\circ} \mathrm{C}$ for further analysis. Analyses were accomplished within 6 months after completion of enrollment by electrochemiluminescence immunoassays using Elecsys 2010 analyzers (Roche Diagnostics, Mannheim, Germany).

Plasma creatinine was measured the day before surgery. Postoperative AKI was graded according to the Kidney Disease Improving Global Outcomes (KDIGO) - creatinine criteria, [12] from maximal postoperative plasma creatinine in relation to the preoperative baseline, and from the need for renal replacement therapy (for grade $3 \mathrm{AKI}$ ). Cardiac surgery was performed with cardiopulmonary bypass $(\mathrm{CPB})$ during moderate hypothermia. Surgical, anesthetic and $\mathrm{CPB}$ management have been described elsewhere $[6,10,11]$. Shortly, general anesthesia was induced with propofol and sufentanil, and before and after $\mathrm{CPB}$ was maintained with remifentanil and sevoflurane. During $\mathrm{CPB}$, anesthesia was maintained with remifentanil and propofol. Perioperative fluid therapy was performed with balanced cristalloid solutions (Sterofundin $\mathrm{ISO}^{\circ}$, B.Braun, Melsungen, Germany) and $6 \%$ hydroxyethyl starch 130/0.4 (Voluven ${ }^{\circ}$ ) (Fresenius Kabi, Bad Homburg, Germany). The $\mathrm{CPB}$ was primed with cristalloid.

\section{Statistical analyses}

Analyses were performed with $\mathrm{R}$ version 3.2.2 (Development Core Team; 2015 R: a language and environment for statistical computing. R Foundation for Statistical Computing, Vienna, Austria. ISBN 3-900051-07-0, http:// www.R-project.org/. Accessed 18 Sept 2016). Data are presented as mean \pm SD if normally distributed or otherwise as median and 25 and $75 \%$ quartiles. Comparisons between groups for univariate predictors of outcome were performed using the two-sided chi-square test for categorical variables and the Mann-Whitney or Kruskal-Wallis test for continuous variables, where appropriate.

The following variables were screened for association with the development of postoperative AKI: age, gender, additive Euroscore, serum creatinine, duration of $\mathrm{CPB}$, duration of surgery, type of surgery, total circulatory 
arrest, preoperative hemoglobin level, preoperative oxygen supplemented cerebral oxygen saturation $\left(\mathrm{ScO}_{2}\right)$, diabetes mellitus, hemofiltration during $\mathrm{CPB}$, plasma GDF-15, high sensitivity troponin T (hsTNT), and Nterminal prohormone of B-type natriuretic peptide (NTproBNP).

The association between the aforementioned variables and the development of CSA-AKI was investigated using logistic regression and machine learning techniques. Model building and variable selection was performed using computer intensive methods (bootstrap aggregation) [14]. In order to investigate nonlinear effects and complex interactions among variables, machine learning methods were utilized (random forests and recursive partitioning using conditional inference trees) [15]. Variable importance (VIMP) and minimal tree depth was used to access the strength of association between each predictor and the development of AKI. Net reclassification improvement and integrated discrimination improvement to assess the additive predictive ability of GDF-15 on the development of AKI were calculated as described by Pencina et al. [16]. Comparisons between receiver-operating characteristic (ROC) curves were performed by the DeLong method and the bootstrap method. The $p$ values (two-tailed) for the DeLong method are presented. Statistical significance was assessed at the $5 \%$ level $(p<0.05$ was considered statistically significant).

\section{Results}

Postoperative AKI was observed in 258 patients (21.9\%) (AKI stage 1 (AKI-1), $\mathrm{n}=175$ (14.9\%); AKI-2, $\mathrm{n}=6$ (0.5 \%); AKI-3, $\mathrm{n}=77(6.5 \%))$. The incidence of AKI-1 and AKI-3 increased significantly from the lowest to the highest tertiles of GDF-15 (Table 1). Accordingly, preoperative plasma GDF-15 was significantly higher in relation to the severity of AKI in comparison with patients without this complication (Fig. 1).

Preoperative, operative, and postoperative characteristics are presented in Table 1, showing that patients in the highest GDF-15 tertile also had a significantly increased risk profile.

The odds ratio from the final logistic regression model, which included GDF-15, was 1.314 (95 \% CI 1.142, 1.551; $p=0.001$ ), with bootstrap reliability of $95.4 \%$. Preoperative plasma GDF-15 was independently and significantly associated with the postoperative development of CSA-AKI. This was not the case for NTproBNP and hsTNT. The full model is presented in Table 2. To further elucidate the effects of adding GDF-15 to the multivariate model, the probability of AKI for a prototype patient is graphically displayed in Fig. 2.

In logistic regression analysis, GDF-15 was comparably and significantly associated with 30-day mortality
(Additional file 1: Table S1). The ROC analysis of the CC-ARF score and the final logistic regression model with and without the inclusion of GDF-15 - as a predictor variable is presented in Fig. $3 a$ and b. In ROC analyses the CC-ARF score had only moderate predictive capacity (area under the curve (AUC) 0.628) for AKI-1 to AKI-3, which was increased by adding GDF-15 (AUC $0.684 ; p<0.001)$. Our logistic regression model investigating the association between preoperative factors and all forms of postoperative AKI (AKI-1 to AKI-3) produced an AUC of 0.738, which was significantly increased $(p<0.001)$ to 0.750 by adding GDF-15 ( $p=$ 0.014).

On analysis restricted to the outcome postoperative AKI-3, the CC-ARF score alone had an AUC of 0.747, which was increased by GDF-15 to $0.795(p<0.001)$. In contrast the AUC of our clinical regression model for AKI 3 was 0.827 , which was improved $(p=0.04)$ to an AUC of 0.848 by incorporating GDF-15. Such improvement in risk stratification was not observed in predicting AKI-1, as the addition of GDF-15 did not improve the AUC for AKI-1 (Fig. 4).

Reclassification analysis revealed that the addition of GDF-15 in the logistic regression model led to a statistically significant increase in the net reclassification ability. The reclassification analysis and tables are presented in Table 3.

Random forest analysis showed that patient age, preoperative creatinine and preoperative plasma GDF-15 were the three most important variables associated with the development of CSA-AKI (Additional file 2: Figure S1). There was significant non-linearity and interactions of predictor variables in the incidence of AKI. As depicted in Additional file 3: Figure S2, within the same additive Euroscore category (panel row), increases in GDF (columns from left to right) are associated with increase in probability for CSA-AKI. As shown, this is especially pronounced in patients with creatinine levels $<180 \mu \mathrm{mol} / \mathrm{l}$. In addition, recursive partitioning revealed the important role of GDF-15 and specific plasma levels of this hormone stratified according to other independent risk factors (Fig. 5).

\section{Discussion}

Several mechanisms mediating a perioperative decrease in renal function have been identified within recent years and several biomarkers have been proposed to facilitate early detection of AKI, i.e., neutrophilgelatinase-associated lipocalin (NGAL), kidney-injury molecule -1 (KIM-1), liver-type fatty acid binding protein (L-FABP), interleukin-18 (IL-18), insulin-like growth factor-binding protein 7 (IGFBP7), and tissue inhibitor of metalloproteinase (TIMP-2) [17]. However, these biomarkers are intended for the early detection of AKI after a 
Table 1 Demographics, preoperative, operative and postoperative characteristics of the patient population according to tertiles of preoperative growth-differentiation factor-15

\begin{tabular}{|c|c|c|c|c|c|}
\hline & GDF tertile 1 & GDF tertile 2 & GDF tertile 3 & Total & $P$ value \\
\hline N & 392 & 392 & 392 & 1176 & \\
\hline Male & $282(71.9 \%)$ & $272(69.4 \%)$ & $256(65.3 \%)$ & 810 (68.9 \%) & 0.129 \\
\hline Age (years) & $59(50 / 67)$ & $69(63 / 74)$ & $72(67 / 77)$ & $68(50 / 74)$ & $<0.001$ \\
\hline NYHA I & $161(41.1 \%)$ & $137(34.9 \%)$ & $113(28.8 \%)$ & $411(34.9 \%)$ & 0.002 \\
\hline NYHA ॥ & $104(26.5 \%)$ & $97(24.7 \%)$ & $87(22.2 \%)$ & $288(24.5 \%)$ & 0.365 \\
\hline NYHA III & $111(28.3 \%)$ & $138(35.2 \%)$ & $146(37.2 \%)$ & $395(33.6 \%)$ & 0.021 \\
\hline NYHA IV & $15(3.8 \%)$ & 19 (4.8 \%) & $44(11.2 \%)$ & 78 (6.6 \%) & $<0.001$ \\
\hline Additive Euroscore & $3(2 / 6)$ & $5(3 / 7)$ & $7(5 / 8)$ & $5(3 / 7)$ & $<0.001$ \\
\hline GDF-15 (ng/ml) & $0.643(0.535 / 0.730)$ & $0.991(0.914 / 1.114)$ & $1.731(1.438 / 2.329)$ & $0.989(0.729 / 1.435)$ & $<0.001$ \\
\hline NTproBNP (pg|ml) & $187.8(74.4 / 495.0)$ & $427.9(155.4 / 900.6)$ & $1044.6(383.4 / 2528.7)$ & $434.5(137.8 / 1139.3)$ & $<0.001$ \\
\hline hsTNT (pg/ml) & $6.3(3.0 / 11.1)$ & $11.4(6.6 / 18.8)$ & $20.4(12.0 / 38.0)$ & $11.4(5.6 / 21.5)$ & $<0.001$ \\
\hline $\mathrm{ScO} 2_{\operatorname{minox}}(\%)$ & $68(63 / 72)$ & $65(60 / 70)$ & $63(57 / 67)$ & $65(60 / 70)$ & $<0.001$ \\
\hline Diabetes mellitus ( $n(\%)$ ) & $152(38.8 \%)$ & $250(63.8 \%)$ & $305(77.8 \%)$ & 707 (60.1 \%) & $<0.001$ \\
\hline LVEF 1 ( $($ (\%)) & $6(1.5 \%)$ & $10(2.6 \%)$ & $24(6.1 \%)$ & $40(3.4 \%)$ & $<0.001$ \\
\hline LVEF 2 (n (\%)) & $57(14.5 \%)$ & $74(18.9 \%)$ & $102(26.0 \%)$ & $233(19.8 \%)$ & $<0.001$ \\
\hline LVEF $3(n(\%))$ & $328(36.4 \%)$ & $307(34.1 \%)$ & $266(29.5 \%)$ & $901(76.8 \%)$ & $<0.001$ \\
\hline Reoperation (n (\%)) & $28(7.1 \%)$ & $35(8.9 \%)$ & $48(12.2 \%)$ & $111(9.4 \%)$ & 0.046 \\
\hline Creatinine $(\mu \mathrm{mol} / \mathrm{l})$ & $73.9(65.1 / 83.6)$ & $82.7(68.6 / 93.3)$ & $93.3(76.6 / 117.0)$ & $81.0(68.6 / 96.8)$ & $<0.001$ \\
\hline eGFR (MDRD) $\left(\mathrm{ml} / \mathrm{min} / \mathrm{m}^{2}\right)$ & $94.2(80.1 / 110.5)$ & $80.7(66.8 / 95.1)$ & $66.2(49.5 / 83.4)$ & $80.9(64.7 / 98.9))$ & $<0.001$ \\
\hline Hemoglobin $(\mathrm{g} / \mathrm{l})$ & $139(130 / 148)$ & $135(125 / 145)$ & $129(115 / 140)$ & $135(124 / 144)$ & $<0.001$ \\
\hline Peripheral vascular disease $(n(\%))$ & $45(11.5 \%)$ & $45(11.5 \%)$ & $47(12.0 \%)$ & $137(11.6 \%)$ & 0.967 \\
\hline CPB time (minutes) & $108(86 / 144)$ & $109(84 / 135)$ & $114(92 / 149)$ & $110(88 / 142)$ & 0.027 \\
\hline $\mathrm{DHCA}(n(\%))$ & 19 (4.8\%) & $8(2.0 \%)$ & $3(0.8 \%)$ & $30(2.6 \%)$ & 0.001 \\
\hline IOP hemofiltration (n (\%)) & $12(3.0 \%)$ & $12(3.0 \%)$ & $36(9.2 \%)$ & $60(5.1 \%)$ & $<0.001$ \\
\hline Isolated CABG (n (\%)) & $173(44.1 \%)$ & $199(50.8 \%)$ & $164(41.8 \%)$ & $536(45.6 \%)$ & 0.033 \\
\hline Mitral valve surgery $(n(\%))$ & $36(9.2 \%)$ & $55(14.0 \%)$ & $83(21.2 \%)$ & $174(14.8 \%)$ & $<0.001$ \\
\hline Aortic valve surgery $(n(\%))$ & $161(41.1 \%)$ & $133(33.9 \%)$ & $161(41.1 \%)$ & $455(38.7 \%)$ & 0.06 \\
\hline MAZE (n (\%)) & $19(4.8 \%)$ & $31(7.9 \%)$ & $53(13.5 \%)$ & 103 & $<0.001$ \\
\hline HDU LOS (days) & $2(2 / 4)$ & $3(2 / 5)$ & $4(2 / 7)$ & $3(2 / 5)$ & $<0.001$ \\
\hline AKI 1 & 37 (9.4 \%) & $53(13.5 \%)$ & $85(21.7 \%)$ & 175 (14.9\%) & $<0.001$ \\
\hline AKI 2 & $3(0.76 \%)$ & $1(0.26 \%)$ & $2(0.51 \%)$ & $6(0.51 \%)$ & 0.65 \\
\hline AKI 3 & $4(1.0 \%)$ & $17(4.3 \%)$ & $56(14.3 \%)$ & $77(6.54 \%)$ & $<0.001$ \\
\hline Renal replacement therapy & $4(1 \%)$ & $17(4.3 \%)$ & $55(14.3 \%)$ & $77(6.45 \%)$ & $<0.001$ \\
\hline 30-day mortality & $2(0.5 \%)$ & $4(1 \%)$ & 17 (4.4 \%) & $23(1.96 \%)$ & $<0.001$ \\
\hline
\end{tabular}

NYHA New York Heart Association grade of heart failure, NTproBNP N-terminal pro-hormone of the B-type natriuretic peptide, hsTNT high-sensitivity troponine-T, LVEF left ventricular ejection fraction (1: $<30 \%$ or severely reduced; $2: 30-50 \%$ or moderately reduced; $3: \geq 50 \%$ or normal), MDRD creatinine clearance according to the Modifications of Diet in Renal Disease formula, CPB cardiopulmonary bypass time, DHCA deep hypothermic circulatory arrest,

IOP-hemofiltration intraoperative hemofiltration during CPB, CABG coronary artery bypass graft, MAZE MAZE - procedure; HDU LOS high-dependency unit time (intensive care and intermediate care unit), $A K I 1$ to $A K I 3$ acute kidney injury according to KDIGO creatinine criteria

renal insult has occurred and not for preoperative risk stratification.

Extending the observations of two recent pilot studies in patients undergoing CABG $[8,9]$, the findings of the present study again show that preoperative plasma GDF15 is an independent predictor of postoperative renal dysfunction in a heterogeneous population of patients undergoing elective cardiac surgery.

GDF-15, also entitled macrophage inhibitory cytokine1 (MIC-1) is a cytokine expressed in many tissues, including myocardium, lung, kidney, brain, liver, and the intestine, upon various stimuli, including myocardial 


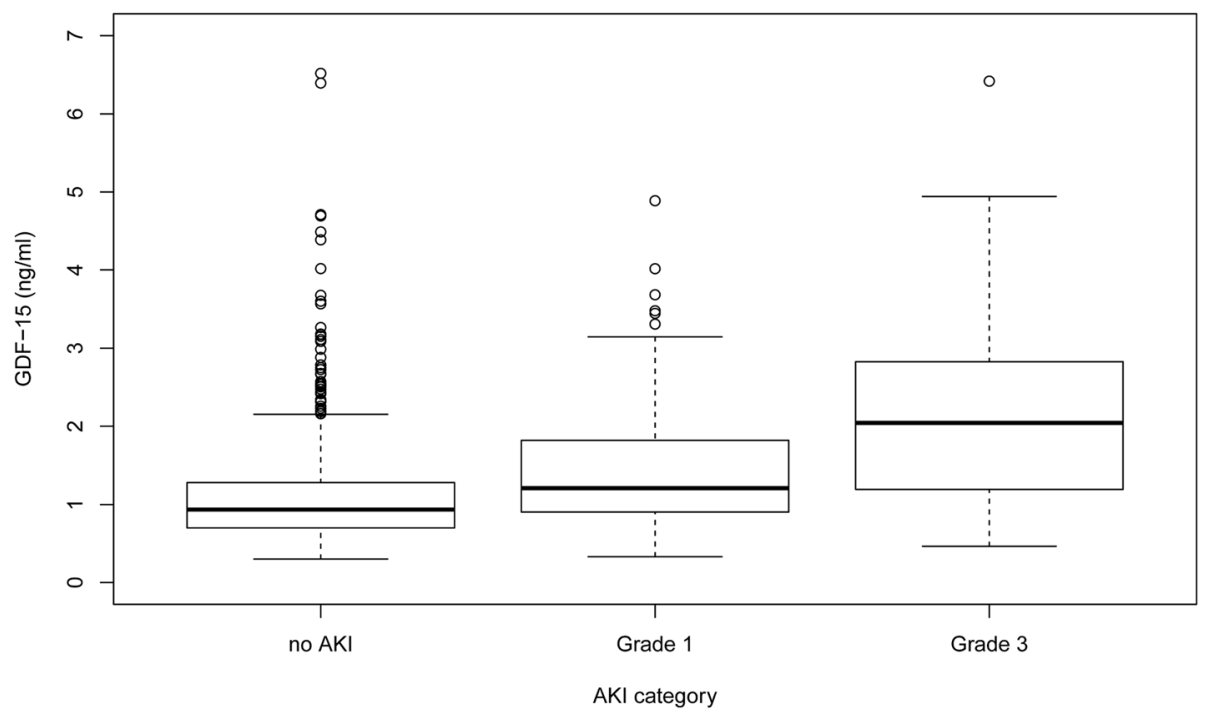

Fig. 1 Plasma growth-differentiation factor-15 (GDF-15) in patients without acute kidney injury (AKI) and in patients with AKI grade 1 (AKI-1) and grade 3: *analysis of variance; $p<0.001$ ). Preoperative plasma GDF-15 was significantly higher in relation to the severity of AKI in comparison with patients without this complication (no AKI vs. Grade 1, $p<0.001$; Grade 1 vs Grade 3, $p<0.001$ )

stretch, volume overload, experimental cardiomyopathy and oxidative stress, other inflammatory cytokines, and ischemia/reperfusion (for a detailed overview see [18]). However, the physiological role of this peptide in the cardiovascular system still remains to be defined.

Our group has recently shown that preoperative plasma GDF-15 is an independent predictor of postoperative mortality and morbidity in patients undergoing cardiac surgery and can further stratify patients beyond the established risk scores such as the Euroscore, and other cardiovascular risk markers such as NTproBNP or hsTNT [10]. The present analysis extends these findings to the prediction of CSA-AKI, an important complication in patients undergoing cardiac surgery, which is associated with poor short-term and long-term prognosis [1].

Employing logistic regression modeling of variables with an established (age, gender, additive Euroscore, serum creatinine, duration of $\mathrm{CPB}$, duration of surgery, type of surgery, total circulatory arrest, preoperative hemoglobin, and diabetes mellitus) or putative $\left(\mathrm{ScO}_{2}\right.$, hemofiltration during ECC, plasma GDF-15, hsTNT, and NTproBNP) role as risk factors for CSA-AKI, we observed that GDF-15 is an independent predictor of CSA-AKI and confirmed this finding using multiple statistical methods. It is of note that in random forest analysis the ability of GDF-15 to predict CSA-AKI was especially pronounced in patients with normal plasma creatinine; one explanation why this hormone had superior predictive ability in comparison with a conventional risk score like the additive Euroscore in our previous study [10]. Additionally, the observation that NTproBNP and hsTNT - despite being widely accepted biomarkers of cardiopulmonary dysfunction - did not predict AKI, further supports the powerful potential of GDF-15 for risk stratification in this regard. It is of note that the risk prediction potential of GDF-15 was primarily related to the ability to predict AKI-3. Whether this

Table 2 Final logistic regression model specification for any grade of acute kidney injury

\begin{tabular}{lclll}
\hline Factor & Odds ratio & $95 \% \mathrm{Cl}$ & $P$ value & Bootstrap reliability \\
\hline Intercept & 0.002 & $0.0006,0.007$ & $<0.001$ & $99.8 \%$ \\
GDF-15 $(\mathrm{ng} / \mathrm{ml})$ & 1.314 & $1.142,1.551$ & $<0.001$ & $95.4 \%$ \\
Age (years) & 1.039 & $1.021,1.058$ & $<0.001$ & $98.9 \%$ \\
Additive Euroscore & 1.074 & $1.009,1.143$ & 0.02 & $71.3 \%$ \\
Creatinine ( $\mu$ mol/l) & 1.007 & $1.002,1.011$ & 0.003 & $79.0 \%$ \\
Diabetes mellitus & 1.362 & $0.981,1.881$ & 0.06 & $50.1 \%$ \\
CPB time (minutes) & 1.006 & $1.003,1.009$ & $<0.001$ & $96.6 \%$ \\
\hline
\end{tabular}

For any increase in growth-differentiation factor-15 (GDF-15) of $1 \mathrm{ng} / \mathrm{ml}$ the odds ratio for developing acute kidney injury of is 1.34 . CPB cardiopulmonary bypass 


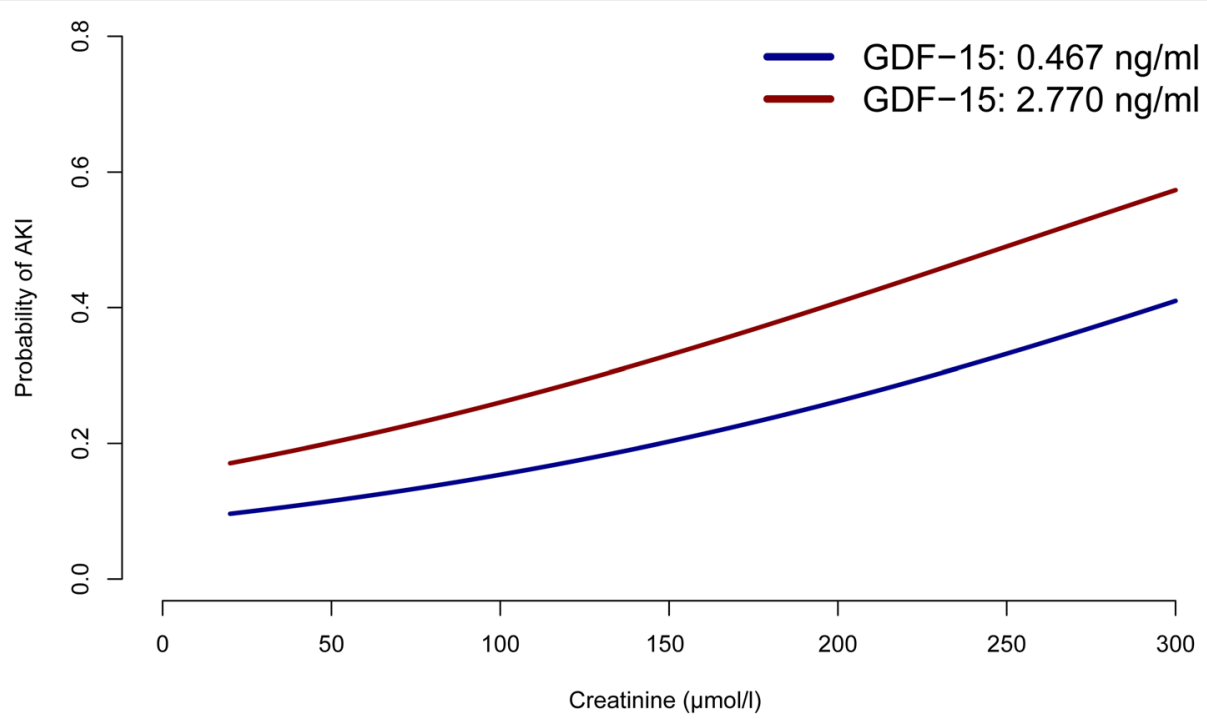

Fig. 2 Probability of a prototypical patient aged 65 years with an additive Euroscore of 5.15, and a duration of cardiopulmonary bypass of 123 minutes for a range of creatinine values ( $x$-axis) and the 5 and $95 \%$ quantiles of growth-differentiation factor- 15 (GDF-15) (0.467 and 2.770, respectively) as derived from the multivariate model. AKI acute kidney injury, CC-ARF Cleveland Clinic Acute Renal Failure (score)

may be related to the physiology or pathophysiology of GDF-15 or that AKI-1 events are very difficult to predict remains speculative.

Various clinical scores for the prediction of renal dysfunction after cardiac surgery have been developed within recent years and these have highly variable predictive ability [7]. We tested the CC-ARF score as one of the most popular scores [13]. As expected, the predictive ability of this score, which was primarily developed to predict postoperative need of dialysis (that renders patients AKI stage 3), was rather poor if used to predict any type of AKI. However, when combined with GDF15 , the predictive ability was markedly improved for any kind of AKI and especially for AKI-3, as the most severe stage of postoperative renal dysfunction. This may have clinical relevance, because the CC-ARF score - in contrast to our model - has been externally validated and is widely used [13].

Very recently, Bignami and coworkers [19] reported that the preoperative plasma level of the endogeneous
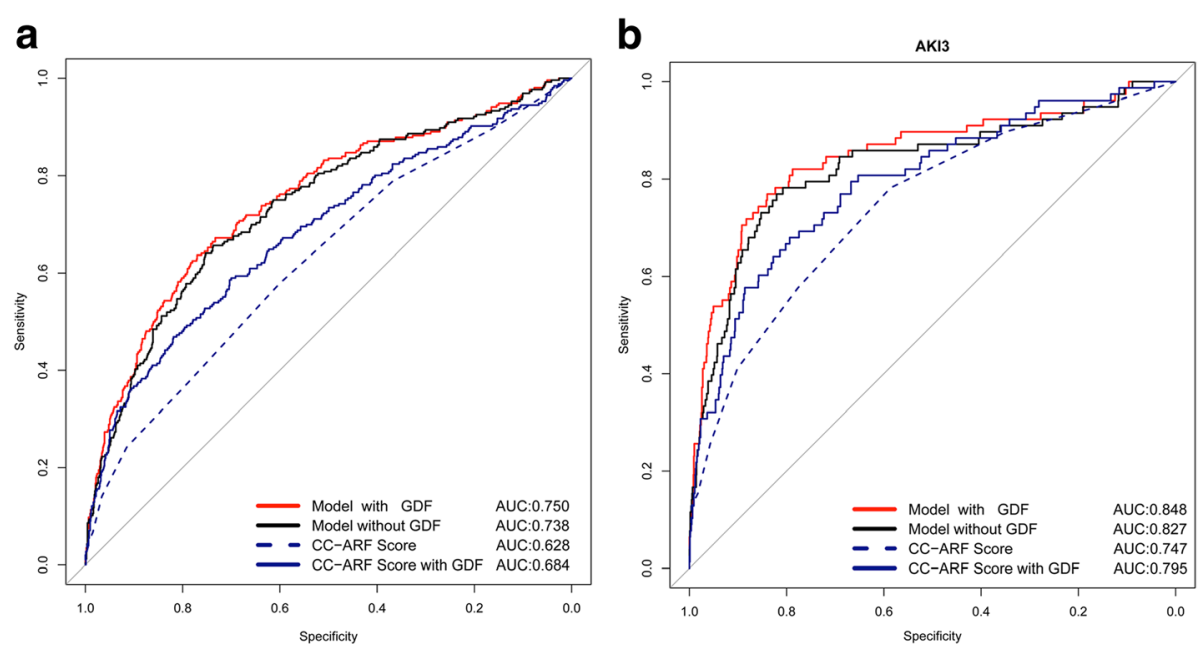

Fig. 3 Receiver operator characteristics of the Cleveland clinic acute renal failure (CC-ARF) score [13] and nested logistic regression models on the development of cardiac-surgery-associated acute kidney injury (CSA-AKI) with and without taking into account preoperative plasma levels of growth-differentiation factor-15 (GDF). a Analyses for any stage of AKI (i.e., AKI-1 to AKI-3). b Analysis restricted AKI-3 only. The inclusion of GDF in the models led to statistically significant (analysis of variance; $p<0.001$ ) predictive ability (either CC-ARF or a model based on additive Euroscore, age, plasma creatinine, diabetes mellitus, and duration of cardiopulmonary bypass). AUC area under the curve 


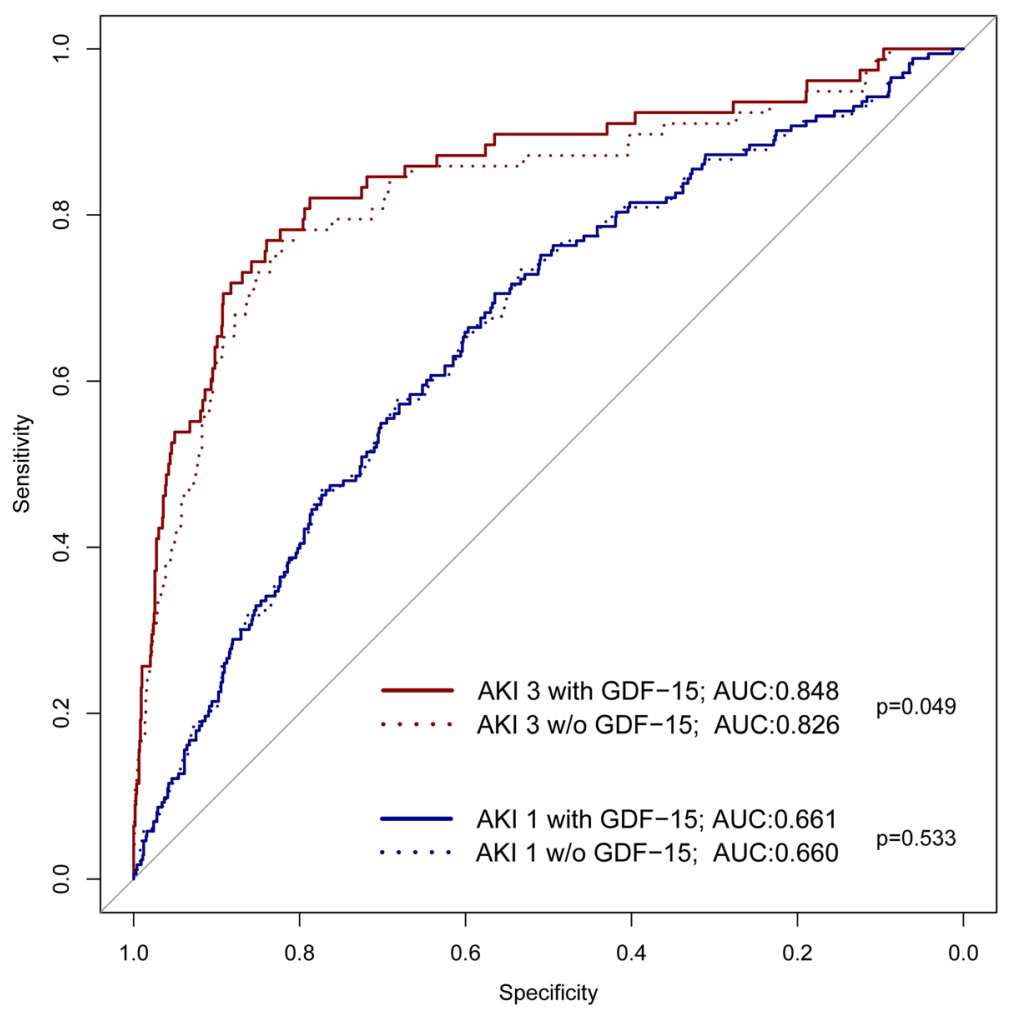

Fig. 4 Receiver operator characteristics of nested logistic regression models on the development of cardiac-surgery-associated acute kidney injury (CSA-AKI) with and without (w/O) taking into account preoperative plasma levels of growth-differentiation factor-15 (GDF) for acute kidney injury (AKI) grade 1 and 3 . While the inclusion of GDF led to a statistically significant increase in the area under curve (AUC) for AKI grade 3, this was not the case for AKI grade 1

hormone ouabain is an independent predictor of AKI in a derivation and a validation cohort of patients undergoing cardiac surgery, and that it improves the predictive ability of a clinical risk score for AKI. It is of note that ouabain and GDF-15 both reflect circulatory stress [20, $21]$, supporting the role of this factor as a trigger of AKI in this setting. But there are also relevant discrepancies between the two peptides. First of all, Bignami et al. provided experimental data for a pathophysiological link between increased circulating levels of ouabain and decreased renal function (i.e., decreased creatinine clearance, increased urinary protein excretion, and reduced podocyte nephrin) [19], whereas a direct detrimental effect of increased GDF-15 levels on renal function has not been shown so far. In contrast, some lines of evidence point to a protective role of GDF-15 in diabetic nephropathy [22].

Additionally, Bignami et al. [19] employed a more rigorous definition of AKI (AKI grade 2 and 3 ) than we did. However, restricting our analyses to AKI-3 we also observed a numerically almost comparable and relevant increase in the AUC in ROC analysis by adding GDF-15 to our logistic regression model. Future studies need to determine which of these peptides has the better power to predict for all stages and the most severe forms of AKI.

\section{Limitations}

The present study has several limitations. First, this is a secondary analysis of a monocentrical, observational study primarily aiming to determine the association between GDF-15 and postoperative morbidity and mortality. Consequently, as we have shown that there is such an association (i.e., that preoperative GDF-15 is an independent marker of morbidity and mortality in this cohort of patients [10]) it cannot be ruled out completely that the described association between preoperative GDF-15 and AKI is epiphenomenal. This may also be true for the observed association of a higher clinical risk profile and GDF-15 tertiles and the observation, that also in this cohort of patients undergoing elective surgery - GDF-15 was an independent predictor of 30-day mortality. With respect to the high mortality in AKI [1], cross-correlation between these two outcomes is almost inevitable. However, the results of the random forest analysis - showing that GDF-15 is especially useful for predicting AKI in patients with low plasma creatinine and who do not typically have a high risk profile - 
Table 3 Reclassification analysis

\begin{tabular}{|c|c|c|c|c|c|}
\hline \multicolumn{6}{|l|}{ Outcome: absent } \\
\hline \multicolumn{6}{|l|}{ Updated model (risk categories) } \\
\hline Initial model (risk categories) & $(0,0.01)$ & $(0.01,0.05)$ & $(0.05,0.1)$ & $(0.1,1)$ & $\%$ reclassified \\
\hline$(0,0.01)$ & 0 & 0 & 0 & 0 & - \\
\hline$(0.01,0.05)$ & 0 & 53 & 2 & 0 & 4 \\
\hline$(0.05,0.1)$ & 0 & 12 & 143 & 13 & 15 \\
\hline$(0.1,1) 0$ & 0 & 24 & & 669 & 3 \\
\hline \multicolumn{6}{|l|}{ Outcome: present } \\
\hline \multicolumn{6}{|l|}{ Updated model (risk categories) } \\
\hline Initial model (risk categories) & $(0,0.01)$ & $(0.01,0.05)$ & $(0.05,0.1)$ & $(0.1,1)$ & $\%$ reclassified \\
\hline$(0,0.01)$ & 0 & 0 & 0 & 0 & - \\
\hline$(0.01,0.05)$ & 0 & 4 & 1 & 0 & 20 \\
\hline$(0.05,0.1)$ & 0 & 1 & 16 & 1 & 11 \\
\hline$(0.1,1)$ & 0 & 0 & 1 & 233 & 0 \\
\hline \multicolumn{6}{|l|}{ Combined data } \\
\hline \multicolumn{6}{|l|}{ Updated model (risk categories) } \\
\hline Initial model (risk categories) & $(0,0.01)$ & $(0.01,0.05)$ & $(0.05,0.1)$ & $(0.1,1)$ & $\%$ reclassified \\
\hline$(0,0.01)$ & 0 & 0 & 0 & 0 & - \\
\hline$(0.01,0.05)$ & 0 & 57 & 3 & 0 & 5 \\
\hline$(0.05,0.1)$ & 0 & 13 & 159 & 14 & 15 \\
\hline$(0.1,1)$ & 0 & 0 & 25 & 902 & 3 \\
\hline
\end{tabular}

Net-reclassification improvement (NRI) (categorical) (95 \% Cl): 0.0229 (0.0014, 0.0445$) ; p$ value 0.03697 . NRI (continuous) (95 \% Cl): 0.308 (0.1739, 0.4421$)$; $p$ value: 0.00001 . Integrated discrimination improvement $(95 \% \mathrm{Cl}): 0.015(0.006,0.024) ; p$ value: 0.00107 . Reclassification table of the model without (initial) and with (updated) growth-differentiation factor-15 (GDF-15) as a predictor of cardiac-surgery-associated acute kidney injury (CSA-AKI). The addition of GDF-15 in the logistic regression model significantly improved prediction of the development of CSA-AKI.

indicates that there are subgroups of patients in whom such an epiphenomenal association is at least not obvious.

As a second point one may argue that the improvements in the clinical models by incorporating GDF-15 were numerically small and despite being statistically significant, they may be of questionable clinical relevance. Nonetheless, the reclassification analyses clearly show that the net effect of reclassification taking into account GDF-15 levels in comparison with the logistic regression model alone was much more pronounced than suggested by the small differences in the AUC [16].

Third, the CC-ARF score had significantly lower ability to predict AKI-1 to AKI-3 and AKI-3 alone than logistic regression analysis based on the present cohort. This contrasts with some studies showing excellent prediction of AKI using the CC-ARF score $[7,13]$ This, however may at least in part be explained by the fact that the present cohort consisted only of patients undergoing elective surgery, as emergency patients were excluded. Thus, the difference between our model and the CC-ARF score may be less pronounced during real-life conditions.
Additionally, we classified AKI only according to creatinine criteria, because data on urine flow were only available for patients studied in 2009. This may lead to discrepancies in comparison with studies analyzing the predictability of renal risk scores based on the analysis of creatinine and urine flow. Recent data from critically ill patients [23] and patients undergoing cardiac surgery [24] clearly suggest that the omission of urine flow may have led to underestimation of the incidence of AKI. However, patients diagnosed with AKI according to creatinine criteria seem to have a much worse prognosis, i.e., higher mortality $[23,24]$. Consequently, AKI diagnosis based only on creatinine criteria may be regarded as more conservative and helpful in identifying those patients with renal dysfunction who have the highest mortality risk.

Fourth, despite confirmation of findings from smaller pilot studies by the present analysis, definitive confirmation of the role of GDF-15 for predicting AKI mandates further and multicenter prospective trials. Ideally, these studies should specifically address diabetes mellitus as a potential confounder, because 


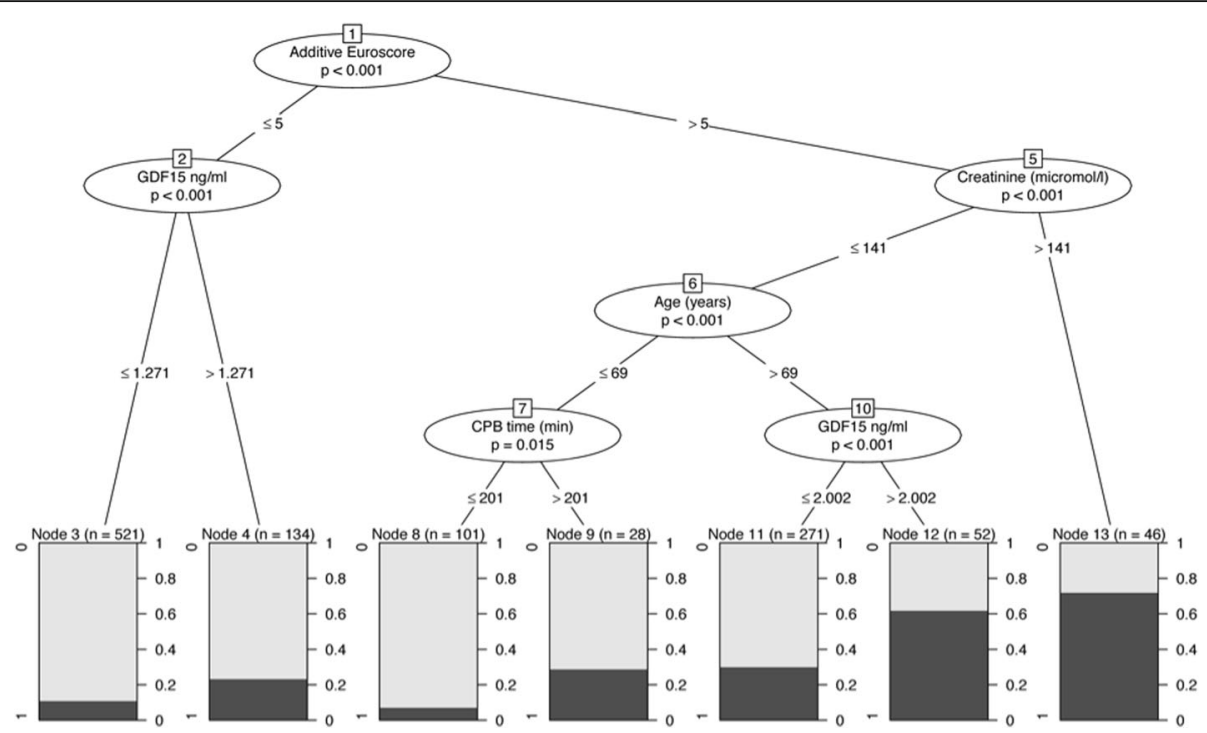

Fig. 5 Recursive partitioning using conditional inference trees. Starting from the top, important variables and their respective cutoffs are presented leading (bottom) to the percentage of patients (within each population partition) developing cardiac-surgery-associated acute kidney injury (CSA-AKI). For example, from the group of patients with additive Euroscore $<5$ and growth-differentiation factor-15 (GDF-15) $<1.271 \mathrm{ng} / \mathrm{ml}, 10 \%$ developed CSA-AKI (Node 3), whereas from the group of patients with additive Euroscore $<5$ and GDF-15 $>1.271 \mathrm{ng} / \mathrm{ml}, 20 \%$ of patients developed CSA-AKI (Node 4). CPB cardiopulmonary bypass

patients with diabetes mellitus also have increased GDF-15 [22] and high risk of AKI after cardiac surgery $[1,5]$. It is of note that the number of patients with diabetes mellitus in the present study in the highest GDF-15 tertile was almost twice as high than in the lowest GDF-15 tertile.

As a last and more general point, it has to be taken into account that a preoperative score or biomarker will never be able to perfectly predict a multifactorial complication like CSA-AKI, because unpredictable intraoperative and postoperative factors, like unexpected prolongation of surgery or prolonged mechanical ventilation [6], may render any prediction model - at least partially - imprecise.

\section{Conclusions}

In conclusion and taking into account the limitations of a monocentric study, but supporting findings from previous work in patients undergoing CABG $[8,9]$, the present analysis shows that preoperative plasma GDF-15 is an independent predictor of postoperative AKI in patients undergoing elective cardiac surgery, and improves the predictive ability of the CC-ARF score as an established renal risk score and of logistic regression models based on the additive Euroscore, age, duration of CPB, and diabetes mellitus. Additionally, this biomarker seems to be particularly helpful for further risk stratification beyond accepted risk factors, i.e., especially in patients with low preoperative creatinine.

\section{Key messages}

- Acute kidney injury (AKI) is a serious and frequent complication in patients undergoing cardiac surgery

- Growth-differentiation factor-15 (GDF-15) is a cytokine expressed upon myocardial stretch and volume overload, and during oxidative stress and ischemia/reperfusion

- GDF-15 has been shown to be reflective of poor prognosis in various clinical settings, including heart failure, myocardial infarction, and in patients undergoing cardiac surgery

- The present study shows that preoperative plasma GDF-15 is an independent predictor of postoperative AKI in patients undergoing elective cardiac surgery and improves the predictive ability of the established renal risk score, the Cleveland Clinic Acute Renal Failure score and of an individual logistic regression model based on the additive Euroscore, age, duration of $\mathrm{CPB}$, and diabetes mellitus

\section{Additional files}

Additional file 1: Logistic regression model specification for 30 day mortality. (DOCX 27 kb)

Additional file 2: Figure S1. Visualization of the predictive ability of each input variable on the development of Cardiac Surgery-associated Acute Kidney injury (CSA-AKI) (random forest model). Variables with greater predictive ability (growth-differentiation factor-15 (GDF-15), age) exhibit high variable importance and low minimal variable depth, whereas 
variables less associated with CSA-AKI (diabetes) exhibit low variable importance and high minimal depth. (TIFF $2787 \mathrm{~kb}$ )

Additional file 3: Figure S2. Covariate plot of the predictions from the random forest model. Four variables are displayed. Within each box, the $x$-axis denotes plasma creatinine $(\mu \mathrm{mol} / \mathrm{l})$, the $y$-axis the probability of developing cardiac-surgery-associated acute kidney injury (CSA-AKI). Within each column of plots, the additive Euroscore increases from bottom to top (legend, right). Within each row of plots the growth-differentiation factor-15 (GDF-15) levels increase from left to right. Significant non-linear interaction takes place between GDF-15 and creatinine. Within each additive Euroscore category (row of plots), increases in GDF-15 (plots more to the right within each row) increase the probability of developing CSA-AKI. However, this effect is more prominent in patients with normal creatinine (x-axis label of each plot). (TIFF $3197 \mathrm{~kb}$ )

\section{Abbreviations}

AKI: Acute kidney injury; AUC: Area under the curve; CABG: Coronary artery bypass graft; CC-ARF: Cleveland Clinic Acute Renal Failure (score);

CPB: Cardiopulmonary bypass; CSA-AKI: Cardiac surgery associated acute kidney injury; eGFR: Estimated glomerular filtration rate (creatinine clearance); GDF-15: Growth-differentiation factor-15; hsTNT: High sensitivity Troponin T; KDIGO: Kidney Disease Improving Global Outcomes; MIC-1: Macrophage inhibitory cytokine-1; NTproBNP: N-terminal prohormone of the B-type natriuretic peptide; $\mathrm{ROC}$ : receiver-operating characteristic; $\mathrm{ScO}_{2}$ : Cerebral oxygen saturation

\section{Funding}

The study was funded by institutional ressources of the Department of Anesthesiology and Intensive Care Medicine, University of Lübeck, Lübeck, Germany. The hormone analyses were kindly performed by Roche Diagnostics, Mannheim, Germany.

\section{Authors' contributions}

The author $\mathrm{MH}$ designed the study, participated in data acquisition and analyses, and wrote the manuscript. $\mathrm{KE}, \mathrm{AEB}, \mathrm{HH}$, and $\mathrm{HP}$ participated in data acquisition, analyses, and preparation of the manuscript. EIC, KE, AEB, and HP participated in the analyses and drafting the manuscript. All authors have read and approved the final manuscript.

\section{Competing interests}

The authors declare that they have no competing interests.

\section{Ethics approval and consent to participate}

The study was approved by the local ethical committee (Ethikkommission der Universität zu Lübeck, AZ: 07-146 and amendment 4 to this study). Written consent was obtained preoperatively.

\section{Author details}

'Department of Anesthesiology and Intensive Care Medicine, University of Lübeck, Ratzeburger Allee 160, D - 23538 Luebeck, Germany. '2Department of Cardiac Surgery, Martin-Luther University, Halle, Germany. ${ }^{3}$ Department of Cardiac Anesthesiology, HELIOS - Clinic, Schwerin, Germany.

\section{Received: 13 April 2016 Accepted: 8 September 2016} Published online: 08 October 2016

\section{References}

1. Stafford-Smith M, Patel UD, Phillips-Bute BG, Shaw AD, Swaminathan M. Acute kidney injury and chronic kidney disease after cardiac surgery. Adv Chronic Kidney Dis. 2008;15:257-77.

2. Heringlake $M$, Knappe $M$, Vargas Hein $O$, Lufft $H$, Kindgen-Milles $D$, Böttige BW, Weigand MR, Klaus S, Schirmer U. Renal dysfunction according to the ADQI-RIFLE system and clinical practice patterns after cardiac surgery in Germany. Minerva Anestesiol. 2006;72:645-54.

3. Pistolesi V, Di Napoli A, Fiaccadori E, Zeppilli L, Sacco Ml, Regolisti G, Tritapepe L, Pierucci A, Morabito S. Severe acute kidney injury following cardiac surgery: short-term outcomes in patients undergoing continuous renal replacement therapy (CRRT). J Nephrol. 2016;29:229-39.
4. Schetz M, Bove T, Morelli A, Mankad S, Ronco C, Kellum JA. Prevention of cardiac surgery-associated acute kidney injury. Int J Artif Organs. 2008:31: 179-89.

5. Bellomo R, Auriemma S, Fabbri A, D'Onofrio A, Katz N, McCullough PA, Ricci Z, Shaw A, Ronco C. The pathophysiology of cardiac surgery-associated acute kidney injury (CSA-AKI). Int J Artif Organs. 2008;31:166-78.

6. Heringlake M, Nowak Y, Schön J, Trautmann J, Berggreen AE, Charitos El, Paarmann $\mathrm{H}$. Postoperative intubation time is associated with acute kidney injury in cardiac surgical patients. Crit Care. 2014;18:547.

7. Kim WH, Lee JH, Kim E, Kim G, Kim HJ, Lim HW. Can we really predict postoperative acute kidney injury after aortic surgery? Diagnostic accuracy of risk scores using gray zone approach. Thorac Cardiovasc Surg. 2015;64(4): 281-9.

8. Kahli A, Guenancia C, Zeller M, Grosjean S, Stamboul K, Rochette L, Girard C, Vergely C. Growth differentiation factor-15 (GDF-15) levels are associated with cardiac and renal injury in patients undergoing coronary artery bypass grafting with cardiopulmonary bypass. PLoS One. 2014;9, e105759.

9. Guenancia C, Kahli A, Laurent G, Hachet O, Malapert G, Grosjean S, Girard C, Vergely C, Bouchot O. Pre-operative growth differentiation factor 15 as a novel biomarker of acute kidney injury after cardiac bypass surgery. Int J Cardiol. 2015;197:66-71.

10. Heringlake M, Charitos El, Gatz N, Käbler JH, Beilharz A, Holz D, Schön J, Paarmann H, Petersen M, Hanke T. Growth differentiation factor 15: A novel risk marker adjunct to the Euroscore for risk stratification in cardiac surgery patients. J Am Coll Cardiol. 2013;61:672-81.

11. Heringlake M, Garbers $C$, Käbler JH, Anderson I, Heinze H, Schön J, Berger KU, Dibbelt L, Sievers HH, Hanke T. Preoperative cerebral oxygen saturation and clinical outcomes in cardiac surgery. Anesthesiology. 2011;114:58-69.

12. Kidney Disease: Improving Global Outcomes (KDIGO) Acute Kidney Injury Work Group. KDIGO Clinical Practice Guideline for Acute Kidney Injury. Kidney Int. 2012;Suppl 2:1-138.

13. Thakar CV, Arrigain S, Worley S, Yared JP, Paganini EP. A clinical score to predict acute renal failure after cardiac surgery. J Am Soc Nephrol. 2005;16: 162-8.

14. Sauerbrei W, Schumache M. A bootstrap resampling procedure for model building: Application to the cox regression model. Stat Med. 1992;11:2093-109.

15. Breiman L. Random forests. Mach Learn. 2001;45:5-32.

16. Pencina MJ, Demler OV. Novel metrics for evaluating improvement in discrimination: net reclassification and integrated discrimination improvement for normal variables and nested models. Stat Med. 2012;31: 101-13.

17. Heringlake M, Schön J, Paarmann H. The kidney in critical illness: how to monitor a pivotal organ system. Best Pract Res Clin Anaesthesiol. 2013;27: 271-7.

18. Unsicker K, Spittau B, Krieglstein K. The multiple facets of the TGF- $\beta$ family cytokine growth/differentiation factor-15/macrophage inhibitory cytokine-1. Cytokine Growth Factor Rev. 2013;24:373-84.

19. Bignami E, Casamassima N, Frati E, Lanzani C, Corno L, Alfieri O, Gottlieb S, Simonini M, Shah KB, Mizzi A, Messaggio E, Zangrillo A, Ferrandi M, Ferrari P, Bianchi G, Hamlyn JM, Manunta P. Preoperative endogenous ouabain predicts acute kidney injury in cardiac surgery patients. Crit Care Med. 2013; 41:744-55.

20. Kempf T, Eden M, Strelau J, Naguib M, Willenbockel C, Tongers J, Heineke J, Kotlarz D, Xu J, Molkentin JD, Niessen HW, Drexler H, Wollert KC. The transforming growth factor-beta superfamily member growth-differentiation factor-15 protects the heart from ischemia/reperfusion injury. Circ Res. 2006; 98:351-60.

21. Goto A, Yamada K, Nagoshi H, Terano Y, Omata M. Stress-induced elevation of ouabainlike compound in rat plasma and adrenal. Hypertension. 1995;26: 1173-6.

22. Adela R, Banerjee SK. GDF-15 as a target and biomarker for diabetes and cardiovascular diseases: a translational prospective. J Diabetes Res. 2015; 490842:14.

23. Wlodzimirow KA, Abu-Hanna A, Slabbekoorn M, Chamuleau RA, Schultz MJ, Bouman CS. A comparison of RIFLE with and without urine output criteria for acute kidney injury in critically ill patients. Crit Care. 2012;16:R200.

24. Lagny GM, Jouret F, Koch NJ, Blaffart F, Donneau AF, Albert A, Roediger L, Krzesinski JM, Defraigne JO. Incidence and outcomes of acute kidney injury after cardiac surgery using either criteria of the RIFLE classification. BMC Nephrol. 2015;16:76. 